\title{
Accelerated Short-Term Techniques to Evaluate Corrosion in TiC Reinforced AA6063 Composites
}

\author{
S.Saravanan ${ }^{1^{*}}$, P.Senthilkumar ${ }^{2}$, T.Palanisamy $^{3}$, M.Ravichandran $^{4}$, \\ V.Anandakrishnan ${ }^{5}$, S.Sankar ${ }^{6}$, A.V.Balan ${ }^{2}$ \\ 1 Department of Mechanical Engineering, St. Joseph's College of Engineering and Technology, Tanjore-624 \\ 204, Tamil Nadu, India. \\ 2 Department of Mechanical Engineering, K.S.R College of Engineering, Tiruchengode-637 215, Tamil Nadu, \\ India. \\ 3 Department of Civil Engineering, K.S.R College of Engineering, Tiruchengode-637 215, Tamil Nadu, India. \\ 4 Department of Mechanical Engineering, Chendhuran College of Engineering and Technology, Pudukkottai- \\ 622507, Tamil Nadu, India. \\ 5 Department of Production Engineering, National Institute of Technology, Tiruchirappalli-620 015, Tamil \\ Nadu, India. \\ 6 Semiconductor Materials and Device Laboratory, Department of Semiconductor Science, Dongguk \\ University - Seoul, Seoul 04620, Republic of Korea. \\ *Corresponding Author: nanosaran007@gmail.com, Tel.: +91-9894648650.
}

\begin{abstract}
AA6063-TiC composites have several weight percentages up to 9 wt. \% were fabricated by using stir casting route method. The effects of the weight percentage of TiC particles on the microstructures and corrosion behavior of AA6063TiC composites were studied. The results revealed that the AA6063-TiC composites exhibited higher density than the AA6063 matrix. The accelerated corrosion tests of AA6063-TiC composites in $3.5 \mathrm{wt} \% \mathrm{NaCl}$ aqueous solution at room temperature, the AA6063-TiC composites have better corrosion resistance than the AA6063 matrix. Increasing the weight percentage of the TiC particles to reduces the corrosion rate of the AA6063-TiC composites. In this process corrosion rate of $0.4402 \mathrm{~mm} /$ year for AA6063 matrix, $0.3891 \mathrm{~mm} /$ year for $3 \mathrm{wt}$. \%, $0.3568 \mathrm{~mm} /$ year for 6 wt. \% and $0.3062 \mathrm{~mm} /$ year for 9 wt. \% of TiC particles respectively. The poor corrosion resistance of the composites can be attributed to the galvanic effects between the AA6063 matrix and TiC reinforcement.
\end{abstract}

Keywords: AA6063-TiC composite, Microstructure, NaCl, Accelerated Corrosion behavior.

\section{Introduction}

Recently, light weight and high strength materials are required for modern automotive industries. Generally, aluminiumbased metal matrix composites play a vital role in aerospace, automotive and military industries such as valves, pumps, cylinder blocks, drives shaft in turbine and helicopter blades used in boat and ship buildings in aggressive environments because due to high corrosion resistance with high tensile properties [1-4]. AA6063 is typically synthesized by several advantages and properties such as excellent casting properties, reasonable strength, corrosion resistance, high strength to weight ratio and heat treatable. AA6063 has been commonly used as base metal for aluminium metal matrix composites reinforced with a variety of reinforcements [5-7]. Corrosion is a slow, rapid deterioration of metals properties such as appearances, surface aspects, and mechanical properties under the influences of the surrounding environment: atmosphere, water, sea water, various solutions, organic environments, etc [8]. Corrosion is the dissolution of a material into its constituent atoms due to chemical reactions with its atmosphere. Alaneme et al. [9] have prepared $\mathrm{AA6063} / \mathrm{Al}_{2} \mathrm{O}_{3}$ composite by adopting two-step stir casting with various percentage of alumina. The corrosion behavior was analyzed different medium such as 5 wt. $\% \mathrm{NaCl}, \mathrm{NaOH}$ and $\mathrm{H}_{2} \mathrm{SO}_{4}$ solution and reported. It exhibited excellent corrosion resistance in $\mathrm{NaCl}$ medium than in the $\mathrm{NaOH}$, and $\mathrm{H}_{2} \mathrm{SO}_{4}$ media. Salman et al. [10] reported at room temperature the comparative electrochemical study of $\mathrm{AZ31}$ and $\mathrm{AZ91}$ magnesium alloys in $1 \mathrm{M} \mathrm{NaOH}$ solutions. When anodized at $3 \mathrm{~V}$ for 30 minutes, the anticorrosion behavior of AZ91 was better than AZ31 which were not anodized. Budruk Abhijeet et al. [11] studied the corrosion behavior of pure magnesium, $\mathrm{Mg}-\mathrm{Cu}$ and $\mathrm{Mg}-\mathrm{Mo}$ composites in $3.5 \% \mathrm{NaCl}$ solution, finding that increasing the volume fraction of reinforcement in both composites and the corrosion rate increased. In similarly volume fraction of reinforcement, molybdenum reinforced composites corroded faster than copper reinforced composites. In accelerated corrosion test, the corrosion degree can be controlled by varying the current density and the time interval of the impressed current. The advantages of adopting this method are achieving a high degree of corrosion within a short period and the easy to control of the corrosion desired.

The objective of this current investigation is to study the accelerated corrosion behavior of AA6063-TiC composites in 3.5 wt. $\% \mathrm{NaCl}$ solution at room temperature. The AA6063-TiC composites were synthesized by using the stir casting route method. The effect of the TiC particles on corrosion characteristics was extensively studied. The marine environment was developed in our laboratory with $3.5 \% \mathrm{NaCl}$ solution.

\section{Experimental Work}

\subsection{Materials and fabrication}

In this study, the matrix AA6063 (Mg-0.56, Si-0.44, Fe- 0.46, Cu-0.02) was selected which is procured in the form of ingots. Titanium carbide is a smart reinforcement for aluminium composites because of its high elastic modulus, grain

5905 | P a g e

February 2017
DOI : 10.24297/jac.v13i10.5898

w w w. cir w o r ld. co m 


$$
\begin{array}{r}
\text { ISSN 2321-807X } \\
\text { Jolume } 13 \text { Number } 10 \\
\text { Journal of Advances in chemistry }
\end{array}
$$

refining effect, high hardness, and wear resistance [12]. It makes a clean and more interface between matrix materials for good wettability achieved [13]. TiC particles size 40-50 $\mu \mathrm{m}$ are used as reinforcement with 3,6 and 9 percentage of weight was used to prepare the composites by stir casting route [14]. AA6063 was melted in a crucible by heating the furnace at $750^{\circ} \mathrm{C}$. The $\mathrm{TiC}$ particles were preheated at $1000^{\circ} \mathrm{C}$ to make their surfaces oxidized. The temperature was first raised above the liquidus temperature of AA6063 near about $750^{\circ} \mathrm{C}$ to melt the AA6063 completely and was then cooled down just below the liquidus to keep the slurry in semisolid state. The preheated TiC was added at this temperature and stirring of the slurry was performed for 3-5 minutes with a motor controlled stirrer. The composite slurry was then heated to $720^{\circ} \mathrm{C}$ and a second stirring performed by a mechanical stirrer to improve the distribution of the TiC particles in the molten of AA6063. The molten composite was then cast into prepared cast iron molds. The prepared different weight percentage sample represented as AA6063-0 wt. \% TiC (sample A), AA6063-3 wt. \% TiC (sample B), AA6063-6 wt. \% TiC (sample C) and AA6063-9 wt. \% TiC (sample D) are shown in Fig. 1.

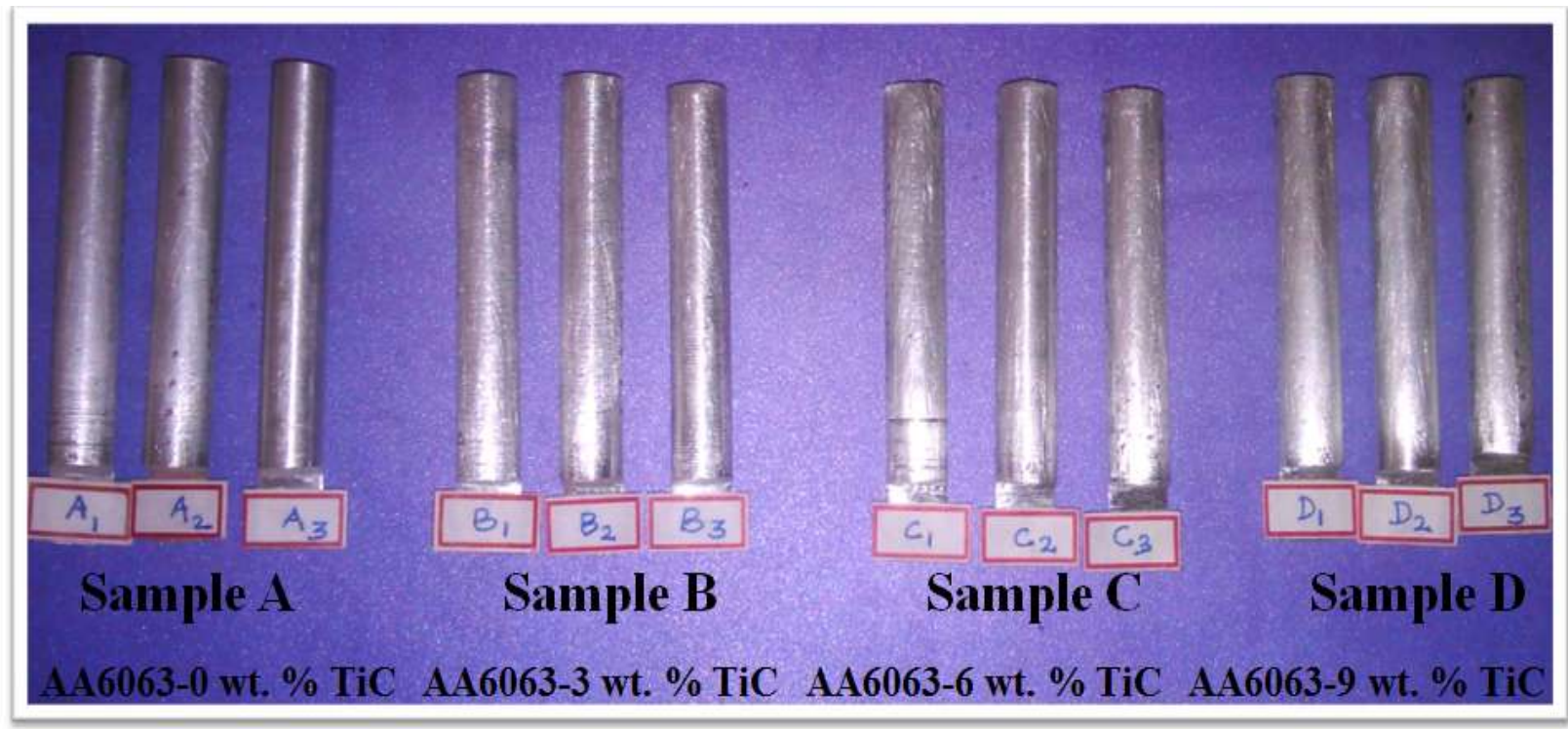

Fig.1. Casted AA6063-TiC composite samples

\subsection{Accelerated Corrosion Test}

The schematic and experimental setups for accelerated corrosion test are shown in Fig. 2 (a) and (b) respectively. It consists of a DC power supply, stainless steel plate, test specimen and the container containing the $3.5 \% \mathrm{NaCl}$ solution. The produced composite cast bars were turned to small rods $10 \times 60 \mathrm{~mm}$ specimen for corrosion test. The composite act as an anode and it's connected to the positive terminal and the stainless steel cylinder act as a cathode, and it's to the negative terminal of the DC power source. Corrosion is initiated by applying a constant voltage (12 V) to the system. The current response is continuously monitored and recorded. Also, the specimens are inspected every 30 minutes visually and measure for the reduction of diameter. The sample surfaces were polished with abrasive sheets and free from the foreign particles. The samples were degreased with acetone and then rinsed in distilled water before immersion in still solutions of $3.5 \% \mathrm{NaCl}$ solution. The solution was prepared by $6500 \mathrm{~cm}^{3}$ of de-ionized water in dissolving $227.5 \mathrm{gm}$ of solid $\mathrm{NaCl}$. In $3.5 \% \mathrm{NaCl}$ solution the specimen was immersed on before weighed and then taken out after $0.5,1,1.5,2$, 2.5 , and 3 hours respectively. After the end of the specified duration, the corrosion deposits on the surface of samples were removed, and then the samples were cleaned with distilled water, rinsed with acetone, dried and weighed. The resultant changes in the weights were noticed. At least three samples were tested, and an average value was taken. Corrosion rate was calculated from weight loss, and it's expressed in mile meter per year (mm/year). In ASTM G31 standard, the mass loss $\left(\mathrm{mg} / \mathrm{cm}^{2}\right)$ of the sample was evaluated by dividing the weight loss by its total surface area $\left(20.41 \mathrm{~cm}^{2}\right)$ [15]. The corrosion rate (weight loss method) was calculated using the following equation 1.

$$
C R=\frac{K \cdot W}{A \cdot D \cdot T}
$$

Where CR is the corrosion rate (mm/year), $\mathrm{K}$ is a constant (87660), $\mathrm{W}$ is the weight loss in the nearest $1 \mathrm{mg}, \mathrm{A}$ is the area $\left(\mathrm{cm}^{2}\right), D$ is the density of the material $\left(\mathrm{g} / \mathrm{cm}^{3}\right)$ and $T$ is the time of exposure $(\mathrm{h})$ to the nearest $0.01 \mathrm{~h}$. 

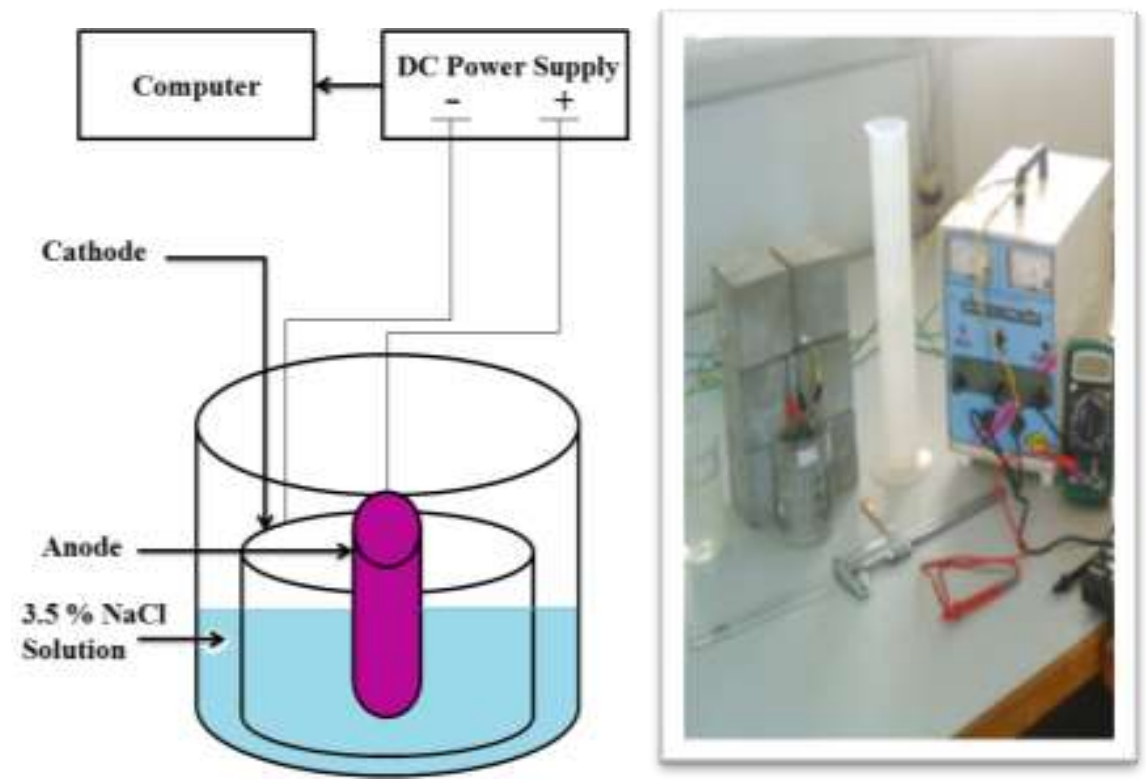

Fig. 2. (a) Schematic set-up (b) Experimental set-up for accelerated corrosion test

\section{Results and discussion}

\subsection{Microstructure Evaluation}

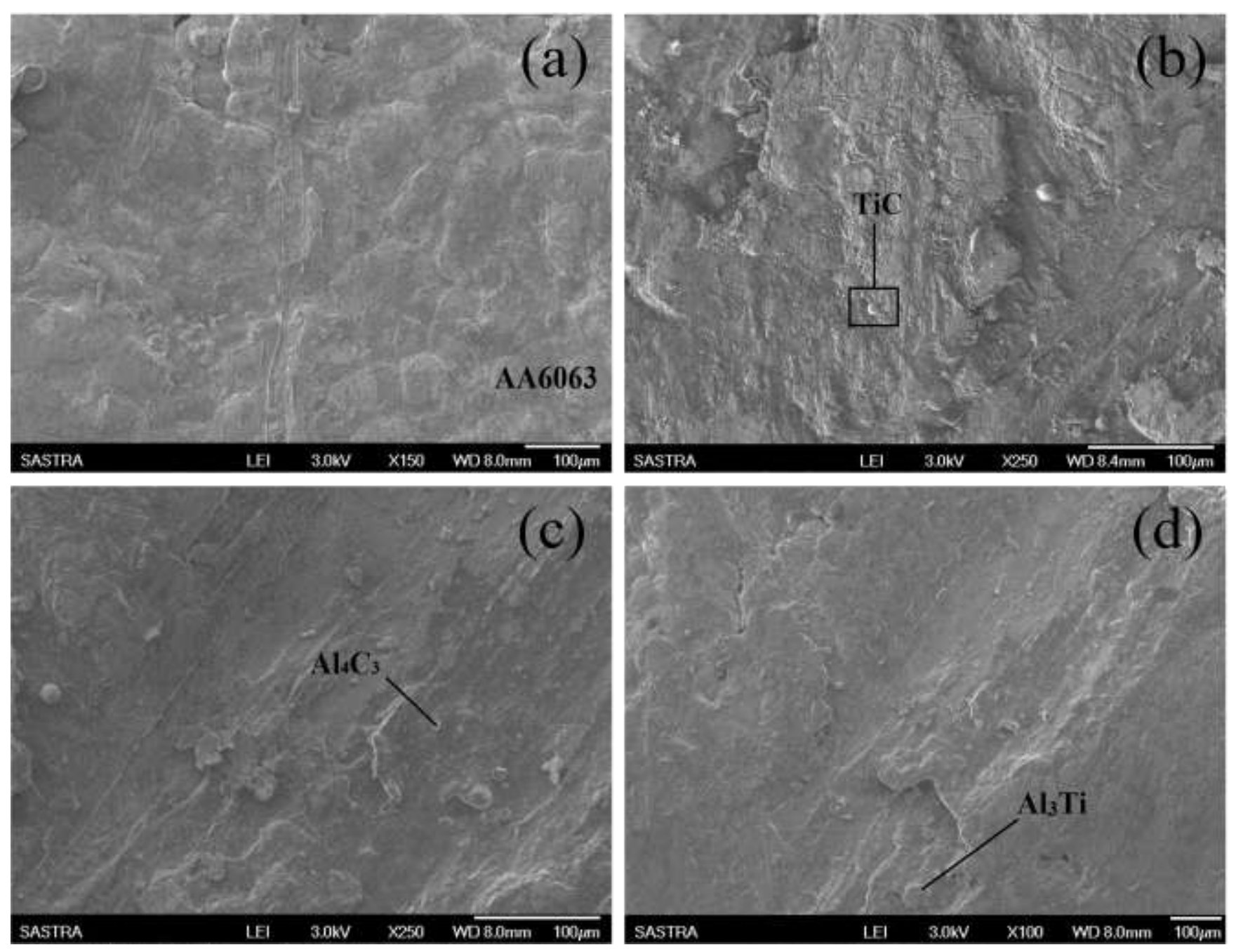

Fig.3. SEM micrographs of AA6063-TiC composites reinforced with TiC particles having of (a) 0 wt. \%, (b) 
3 wt. \%, (c) 6 wt. \% and (d) 9 wt. \%

Fig. 3 shows the SEM images of samples A, B, C and D before the accelerated corrosion test. With respect the SEM images of specimen $A, B, C$ and $D$, it is ensured that the integrity of particles in materials matrix is due to plastering AA6063 by TiC particles. It has been observed that increasing the weight percentage increases the agglomerations of the $\mathrm{TiC}$ particles. The TiC particles are reacting with aluminium to give intermetallic compounds like $\mathrm{Al}_{4} \mathrm{C}_{3}$ and $\mathrm{Al}_{3} \mathrm{Ti}$ are conforms and marked in SEM graphs (Fig. 3 (c) and (d)) and respected to the equation 2.

$$
13 \mathrm{AI}+3 \mathrm{TiC} \rightarrow 3 \mathrm{Al}_{3} \mathrm{Ti}+\mathrm{Al}_{4} \mathrm{C}_{3}
$$

The intermetallic components of AA6063-TiC composites have high electric conducting properties [21]. The AA6063-TiC composites contain $0,3,6$ and $9 \mathrm{wt}$ \% of TiC particles exhibited densities $2.605,2.682,2.79$ and $2.842 \mathrm{~g} / \mathrm{cm}^{3} \mathrm{respectively}$ and showed AA6063-TiC composites having higher densities than the pure AA6063 matrix. It has shown that increasing the weight percentage of TiC particles increases the density of the composites.

\subsection{Corrosion behavior of the AA6063-TiC composites}

The decrease in corrosion resistance with $\mathrm{TiC}$ reinforcement in $\mathrm{Al}-\mathrm{Cu}$ and $\mathrm{Al}-\mathrm{Mg}$ matrices due to the easier breakdown of a passive oxide film in the voids at interfaces has been reported [16,17]. In the immersion of $3.5 \% \mathrm{NaCl}$ solution for AA6063-TiC composite has involving the following reactions (equation 3 - 9). In the cathodic reaction, electrons are produced by the anodic reaction in which the Al dissolves under the assertiveness action of the chloride ions. The anodic branch, the aluminium surface develops an oxide layer. It gets destroyed with increasing the applied potential at the most negative values in the presence of the corrosive $\mathrm{Cl}$ species. A salt barrier is formed within the pits in their formation. In this case, $\mathrm{AlCl}_{3}$ which could then form $\mathrm{AlCl}_{4}{ }^{-}$and the chloride ions do not enter into the formed oxide layers, but they are chemisorbed onto the oxide surface. It acts as a reaction partner, aiding the oxide to dissolve via the formation of oxychloride complexes.

$$
\begin{aligned}
& \mathrm{NaCl} \rightarrow \mathrm{Na}^{+}+\mathrm{Cl}^{-} \\
& 2 \mathrm{H}_{2} \mathrm{O}+2 \mathrm{e}^{-} \rightarrow \mathrm{H}_{2}+2 \mathrm{OH}^{-} \\
& \mathrm{Al} \rightarrow \mathrm{Al}_{3}^{+}+3 \mathrm{e}^{-} \\
& \mathrm{Al}+2 \mathrm{H}_{2} \mathrm{O} \rightarrow \mathrm{AlO}_{2}^{-}+4 \mathrm{H}^{+}+3 e^{-} \\
& \mathrm{Al}+3 \mathrm{H}_{2} \mathrm{O} \rightarrow \mathrm{Al}(\mathrm{OH})_{3}+3 \mathrm{H}^{+}+3 e^{-} \\
& \mathrm{Al}+4 \mathrm{Cl}^{-} \rightarrow \mathrm{AlCl}_{3}^{-}+\mathrm{HCl} \\
& \mathrm{AlCl}_{3}{ }^{-}+3 \mathrm{H}_{2} \mathrm{O} \rightarrow \mathrm{Al}(\mathrm{OH})_{3} \mathrm{Cl}_{3}
\end{aligned}
$$

The above reaction results in activation of the aluminium crystallite boundary so that crystallites break off from the film and become very resolvable. The thickness of the film decreases until local defects are formed. The $\mathrm{Cl}^{-}$ions erode the exposed surface of AA6063 matrix easily. Higher the $\mathrm{Cl}^{-}$ion concentration, easier the complexation reaction occurs. The aluminium corrosion potential was more negative to increase the $\mathrm{Cl}^{-}$ion concentration and tends to be more sensitive to pitting corrosion. It is understood that competitive adsorption promotes $\mathrm{Cl}^{-}$ions as a substitute of oxide on the aluminium surface so that pitting corrosion gets induced. But the competitive adsorption theory cannot explain satisfactorily the dissolving mechanism of aluminium the oxide film. According to complexation corrosion theory, a compact $\mathrm{Al}_{2} \mathrm{O}_{3}$ film is expected to form on the aluminium surface in neutral chloride medium offering corrosion resistance. The $\mathrm{Cl}^{-}$ions are adsorbed selectively on the crystal lattice of the oxide hydrate film under the effect of electric field. The AA6063 and composites contain the $\mathrm{Al}-\mathrm{Fe}-\mathrm{Si}$ and $\mathrm{Mg}_{2} \mathrm{Si}$ intermetallic phases.

The elements in these intermetallic phases undergo oxidation to yield their respective oxides such as $\mathrm{SiO}_{2}, \mathrm{FeO}$, and $\mathrm{MgO}$ which reduce the corrosion rate in composites by decreasing the micro galvanic coupling between conducting intermetallic phases and the matrix in composites. In these $\mathrm{Al}$ alloys, a presence of intermetallics such as $\mathrm{Ti}_{3} \mathrm{Al}, \mathrm{Ti}_{3} \mathrm{AlC}, \mathrm{Al}_{3} \mathrm{Ti}$, and $\mathrm{Ti}_{3} \mathrm{Cu}$ which are cathodic on metal matrix has been reasoned to be the contributing factor the enhanced pitting corrosion. The superior passivation potential exhibited by the TiC reinforced composites is attributable to the reduction in the pit dissolution kinetics by the oxides of metals. The TiC particles react with aluminium to give intermetallic compound like $\mathrm{Al}_{4} \mathrm{C}_{3}$. Thus, the corrosion resistance is enhanced in the case of $\mathrm{TiC}$ reinforced $\mathrm{Al}$ composites.

Fig. 4 shows the variation in the weight loss with time of tested specimens immersed in $3.5 \% \mathrm{NaCl}$ solutions. It has been found that the AA6063-TiC composites showed better weight losses when compared with the pure AA6063 matrix. It is observed in this case that weight loss decreased with the increase in weight percentage of TiC. AA6063 has the highest 
weight loss percentage (65\%) and the AA6063-9 wt. \% of TiC (56\%) had the least weight loss followed by the AA6063-6 wt. \% TiC composite (59\%). When increasing the percentage of TiC to the AA6063-TiC composites, the weight loss gradually decreased, due to the uniform distribution of $\mathrm{TiC}$ particles. The formation of a stable passive layer of $\mathrm{Al}(\mathrm{OH})_{3}$, which is formed of the aluminium alloy and the $\mathrm{Al}(\mathrm{OH})_{3}$ protective oxides formation of such oxides results in less corrosion reaction over a period. Saturation of the solution with anodic ions and also the formation of a relatively more stable passive oxide layer, a steady state condition were arrived after few days irrespective of the materials. When compared with the AA6063 matrix, the AA6063-TiC composites show a decrease in the weight loss.

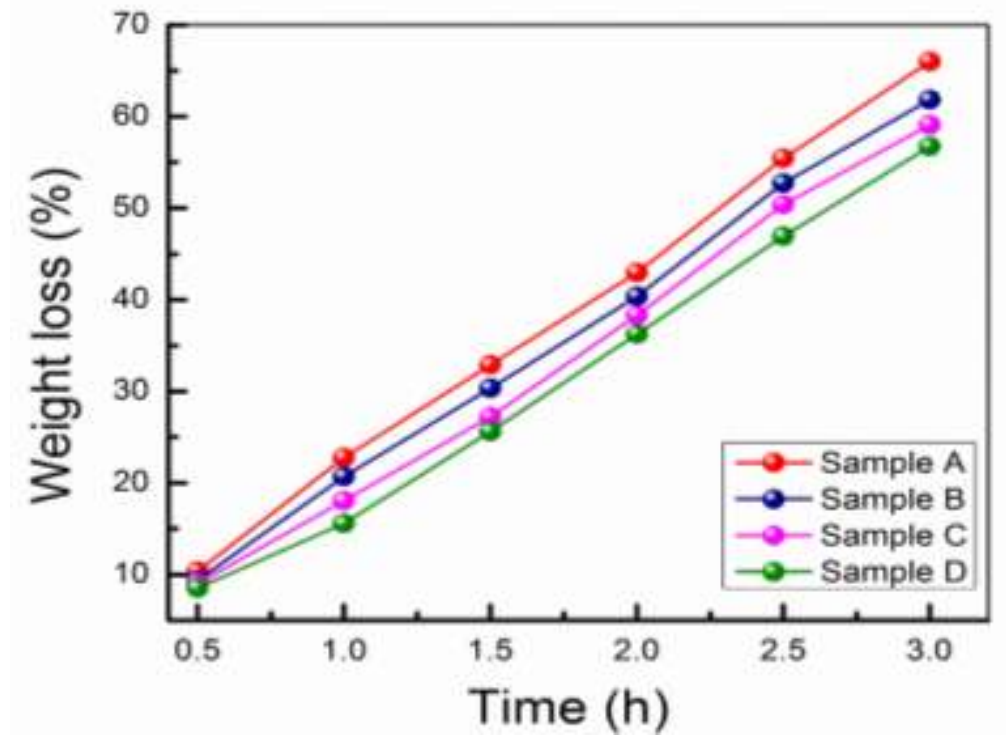

Fig.4. Variation in weight loss with time for AA6063-TiC composites after accelerated Corrosion test in

\section{$3.5 \% \mathrm{NaCl}$ solution.}

Fig. 5 shows the variation in the corrosion rate of AA6063-TiC composites with the time in $3.5 \% \mathrm{NaCl}$ solution. It has been found that the AA6063-TiC composites showed better corrosion resistance when compared with the AA6063 matrix. Increasing the weight percentage of the TiC particles increasing the corrosion resistance of the AA6063-TiC composites. The corrosion rate of AA6063 was greater than that of the AA6063-TiC in 3.5\% NaCl medium. From that observation, the corrosion resistance of the materials under examination increases as the time is increased. The phenomenon of gradually increasing corrosion resistance is due to the formation of passive films on a surface of the AA6063-TiC composite. The developments of the corrosion resistance of the AMCs due to the increase in the TiC volume fraction were reported by many researchers [18, 19, 20]. For example, Anandamurthy et al. [21] examined the effects of TiC reinforcements and the concentration of $\mathrm{Cl}$ ions in solution on the localized corrosion characteristics of AA6063-TiC composites. They reported that the increase in weight percentage of $\mathrm{TiC}$ reinforcement in the AA6063-TiC composites resulted in a significant decrease in pitting potential.

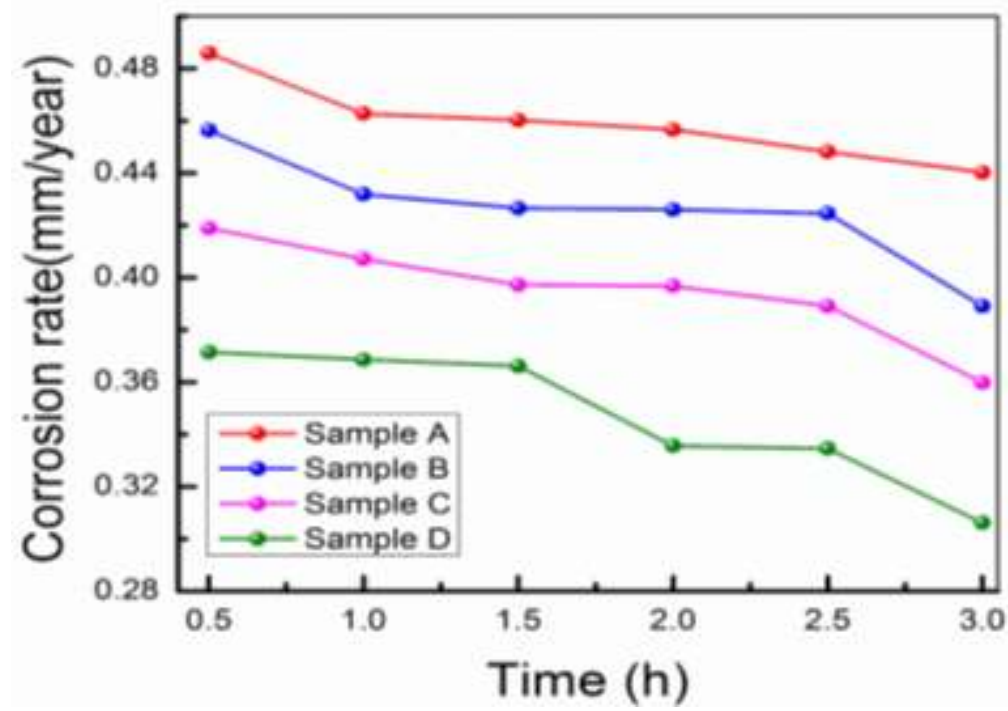

Fig.5. Variation in corrosion rate with time for AA6063-TiC composites after accelerated corrosion test in $3.5 \% \mathrm{NaCl}$ solution. 
Fig. 6 shows the variation in the temperature with respect to time in the accelerated corrosion test in $3.5 \% \mathrm{NaCl}$ solution. It has been found that the AA6063-TiC composites showed a lower amount of heat generated when compared with the AA6063 matrix. Increasing the weight percentage of the TiC particles to decreasing the temperature of the AA6063-TiC composites due to the least amount of $\mathrm{Al}(\mathrm{OH})_{3}$ formation. In every 30 minutes of time intervals, the temperature linearly decreases with the addition of $\mathrm{TiC}$ particles for different weight percentages. With the increase in immersion time the specimen surfaces become gradually covered by aluminium hydroxide likely say clusters of corrosion product. Effect of temperature on the corrosion rate of AA6063-TiC composites depends on the energy activation of corrosion. The corrosion rate decreases more rapidly at lower temperatures than higher temperatures for AA6063-TiC composites.

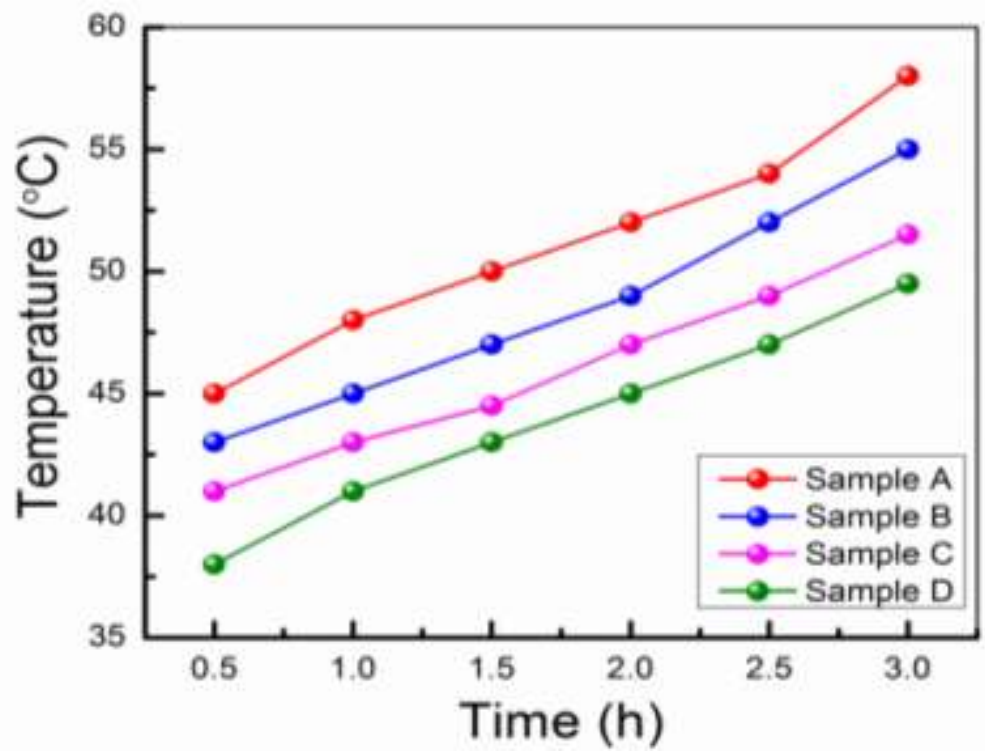

Fig.6. Variation in temperature with time for AA6063-TiC composites after accelerated corrosion test in $3.5 \% \mathrm{NaCl}$ solution

Fig. 7 displays the current variations with respect to time in the accelerated corrosion test in $3.5 \% \mathrm{NaCl}$ solution. Initially, it can be seen that some relatively small amplitude variations are encountered, but after that, the later stage, an increase in current occurs. It is considered that this corresponds to the addition of TiC to a corresponding increase in electrical resistance of AA6063-TiC composites. The stable of the corrosion products layer, and, thus, a decrease in the localized current density; once the corrosion product is built, the current density increases. AA6063-TiC composite pitting corrosion is localized accelerated dissolution that breaks down the protective passive film on the surface. Breakdown to the film is due to the presence of $\mathrm{Cl}^{-}$ions and in aerated aqueous solutions. This is also influenced by an electrolyte within 4.5 to $8.5 \mathrm{pH}$ value.

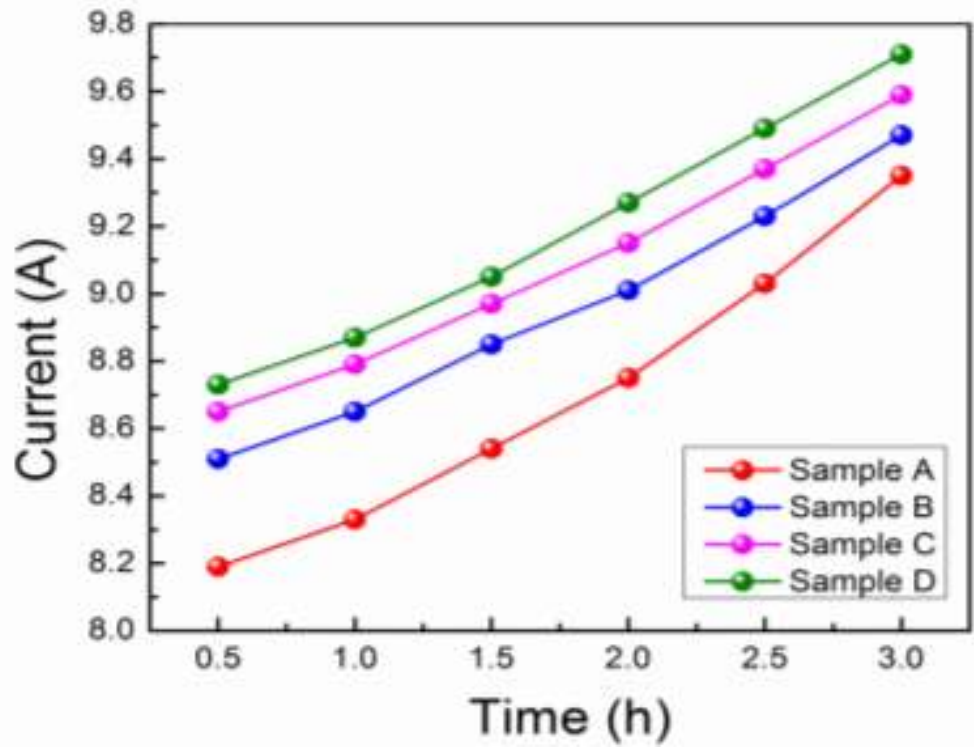

Fig.7. Variation incurrent with time for AA6063-TiC composites after accelerated corrosion test in $3.5 \%$ $\mathrm{NaCl}$ solution 
In Fig. 8 indicates the $\mathrm{pH}$ of the solution measured in AA6063 pits was found to be 4 to 6 by using a freezing method when the $\mathrm{pH}$ of the bulk solution was 11. Corrode in acidic solution due to forming $\mathrm{Al}_{3}{ }^{+}$and formation of $\left(\mathrm{AlO}_{2}{ }^{-}\right)$in alkaline solution. Alodan et al. [22] have investigated corrosion of aluminium alloys by microscopy techniques. They reported that chloride solutions attack AA6061 through the $\mathrm{Mg}, \mathrm{Si}$, and $\mathrm{Al}$ inclusions in the early stages, and the partly dissolve and form corrosion products. During the exposure to a chloride solution, $\mathrm{Mg}$ selectively dissolves. However, the $\mathrm{pH}$ of the bulk solution was rather, and small changes in the $\mathrm{pH}$ might not be noted. Fig. 9 shows the diameter reduction versus length of tested specimens immersed in $3.5 \% \mathrm{NaCl}$ solutions. The corrosion process is under a control of adsorption of chloride ions at the AA6063-TiC composite/ $\mathrm{NaCl}$ solution interface. The diameter of the specimen was decreased as time elapsed, indicating an increase in the corrosion rate with time and the nonproductive nature of the corrosion products.

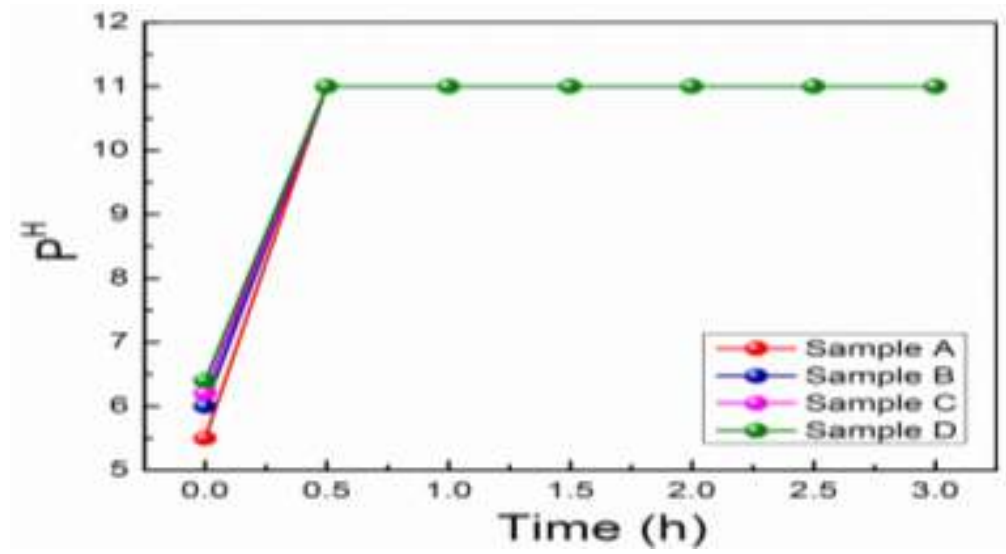

Fig.8. Variation in pH with time for AA6063-TiC composites after accelerated corrosion test in $3.5 \% \mathrm{NaCl}$ solution.

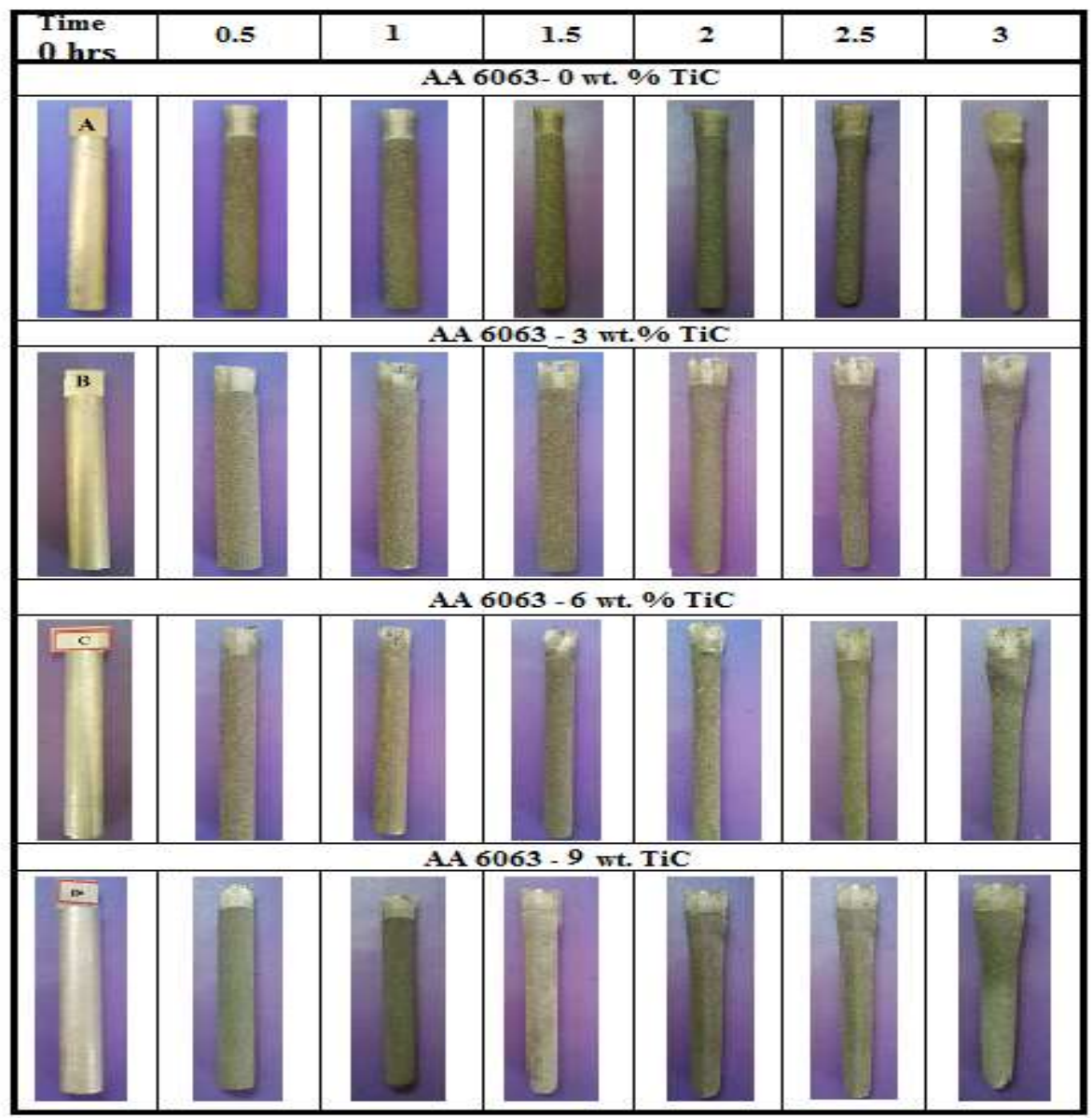

Fig.9. Variation in diameter with time for AA6063-TiC composites after accelerated corrosion test in $3.5 \%$ $\mathrm{NaCl}$ solution. 


$$
\begin{array}{r}
\text { ISSN 2321-807X } \\
\text { Jolume } 13 \text { Number } 10 \\
\text { Journal of Advances in chemistry }
\end{array}
$$

Fig. 10 (a) shows typical SEM micrographs of the corroded surface for sample A after immersion in $3.5 \% \mathrm{NaCl}$ solution for $3 \mathrm{~h}$ at room temperature. It is clear that severe damage was found on the surface, and large pits are visible on the surface, indicating susceptibility of the material towards pitting corrosion in $\mathrm{NaCl}$ medium. The surface of sample $\mathrm{A}$ undergoes severe degradation, especially along the grain boundaries. These grain boundaries provide privileged corrosion initiation sites because of the discontinuity in the surface due to the change in structure. In contrast, the sample B, C, and D (Figs.10 (b), (c) and (d)) corroded surfaces exhibited less damage than the sample A. In addition to grain boundary attack; pitting occurred at the sites where the TiC particles agglomerate. The better corrosion resistance of AA6063-TiC composites compared with the AA6063 matrix may attribute to the fact that TiC particles are being ceramics and remain inert in the $\mathrm{NaCl}$ solution. They are hardly affected by the $\mathrm{NaCl}$ aqueous medium. Even if the corrosion rate of the AA6063-TiC composites is less with the formation of pits on the surface. However, the addition of TiC particles both the uniform corrosion rate of the AA6063 and the resistance towards the pitting type of corrosion. Some evidence of a galvanic effect between the AA6063 matrix and the TiC particles are found. The TiC particles resist the cruelty of the medium attack to a certain extent. Moreover, there is evidence for the presence of grain boundary corrosion and pitting corrosion in the AA6063-TiC composites (Fig. 10 (b)). Hence the nature of the interfacial bond is critical in the corrosion process. It is believed that the improvement of the corrosion resistance of the AA6063 matrix, observed in the present investigation due to the good interfacial bonding between the AA6063 and TiC particles. The increase in corrosion resistance with increasing the weight percentage of TiC particles also supports this.
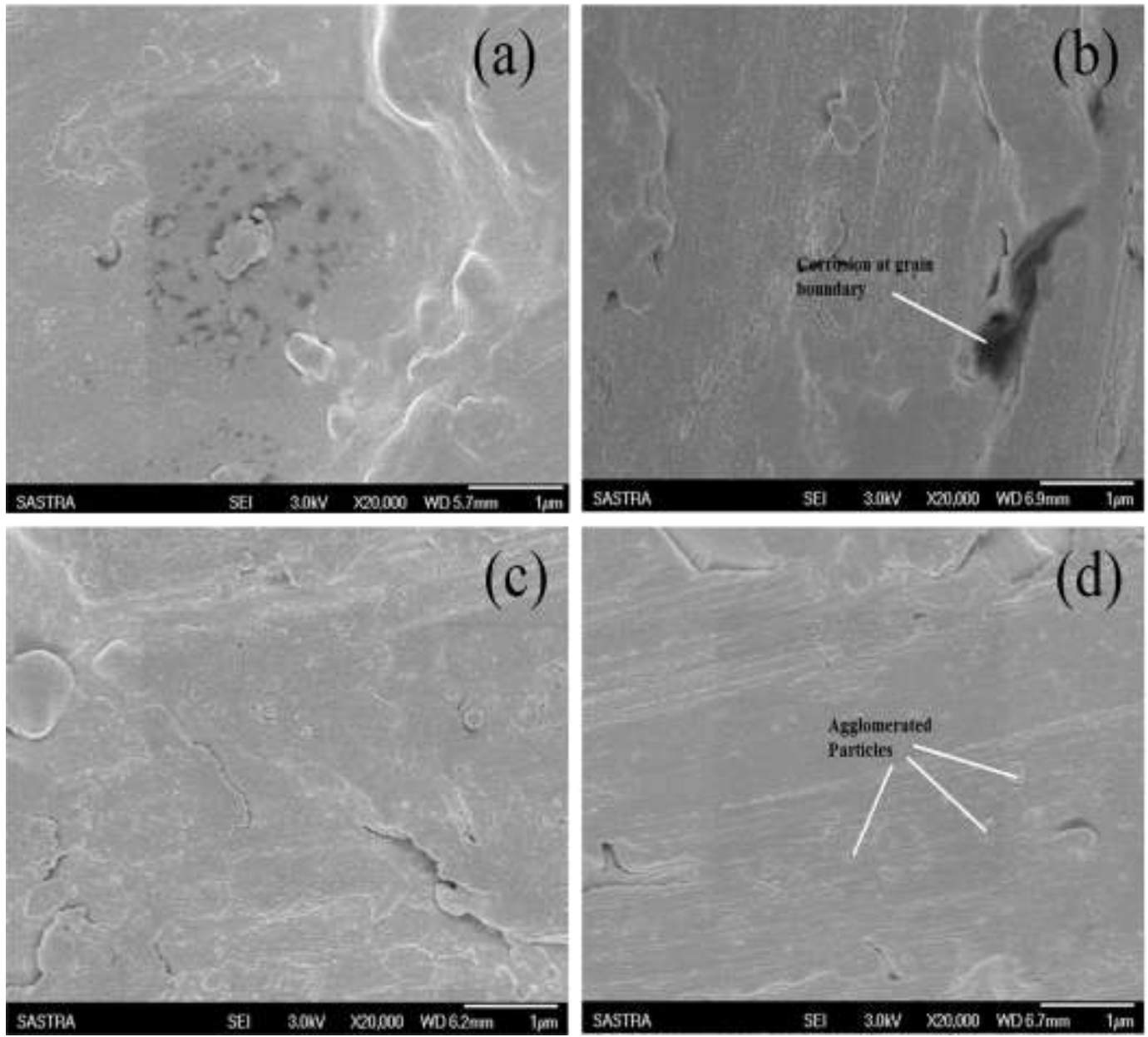

Fig. 10. SEM micrographs of corroded surface of AA6063-TiC composites reinforced with TiC particles having of (a) 0 wt. \%, (b) 3 wt. \%, (c) 6 wt. \% and (d) 9 wt. \%.

\section{Conclusion}

In this investigation, the following conclusions have been drawn.

- In accelerated corrosion test, the AA6063-TiC composites exhibited better corrosion resistance than the AA6063matrix in $3.5 \% \mathrm{NaCl}$ solution.

- Increasing the weight percentage of the TiC particles decreased the corrosion rate of the AA6063-TiC composites. Moreover, the uniform sized TiC particles are enhanced to the corrosion resistance of the AA6063-TiC composites.

- The extent of corrosion resistance was increased with increasing TiC which may be due to increasing in bonding strength.

- The corrosion resistance decreases with a decrease in temperature, increase in time and increase in current. 


\section{Acknowledgements}

The authors would like to thank the Mr. S.Ramesh, Department of Mechanical Engineering, KSR College of Engineering, Tiruchengode, India for providing necessary support in conducting the experiments.

\section{References}

[1]. Surappa, M K. 2003. Aluminium matrix composites challenges and opportunities, Sadhana 28: Parts 1 \& 2. 319334.

[2]. Ravichandran, M. NaveenSait, A. and Anandakrishnan, V. 2014.Al-TiO2-Gr powder metallurgy hybrid composites with cold upset forging. Rare Metals. 33:6, 686-696.

[3]. Herman Pratikno. 2015. Aging treatment to increase the erosion-corrosion resistance of AA6063 alloys for marine application. Procedia Earth and Planetary Science. 14, 41-46.

[4]. Babu Rao, J. Venkatarao, D. Narasimhamurthy, I. and NRMR Bhargava. 2011. Mechanical properties and corrosion behavior of fly ash particles reinforced AA2024 composites. Journal of Composite Materials. 46(12), 1393-1404.

[5]. Ravichandran M and Anandakrishnan V.2016. Hot upsetting studies on sintered (Al-TiO2-Gr) powder metallurgy hybrid composite, Strength of Materials. 3, 135-146.

[6]. Ashok Kumar Srivastava, Karabi Das, and Sandeep Kr. Toor. 2015. Corrosion behavior of TiC reinforced hadfield manganese austenitic steel matrix in-situ composites. Open Journal of Metal. 5, 11-17.

[7]. Ravichandran, M. Manikandan, A.R., Omkumar, M.S. 2016. Investigations on Properties of Al-B4C Composites Synthesized through Powder Metallurgy Route. Applied Mechanics and Materials. 852, 93-97.

[8]. Hosni Ezuber, A El-Houd, and F El-Shawesh. 2008. A study on the corrosion behavior of aluminium alloys in seawater. Materials and Design. 29, 801-805.

[9]. Alaneme, K K. and Bodunrin, M O. 2011. Corrosion behavior of alumina reinforced aluminium (6063) metal matrix composites. Journal of Minerals and Materials Characterization and Engineering. 10(12), 1153-1165.

[10]. Salman, S A. Ichino, R. and Okido, M. 2010. A Comparative electrochemical study of AZ31 and AZ91 magnesium alloys. International Journal of Corrosion. 1-7.

[11]. Budruk Abhijeet, S. Balasubramaniam,R. and Gupta, M. 2008. Corrosion behaviour of Mg-Cu and Mg-Mo composites in 3.5\% NaCl. Corrosion Science. 50 (9), 2423-2428.

[12]. Bauri, R. Yadav, D. and Suhas G.2011. Effects of friction stir processing (FSP) on microstructure and properties of Al-TiC in situ composite. Materials Science Engineering. A(05) 528, 4732-4739.

[13]. Leon, C A. Lopez, V H. Bedolla, E and Drew R A L. 2002. Wettability of TiC by commercial aluminium alloys. Journal of Materials Science. 37(16), 3509-3514.

[14]. Kaviya, K. Saravanan, S. Ravichandran, M. and Senthilkumar, P. 2015. Microstructural Analysis of AA6063-5 and 7.5 wt. \% TiC Nanocomposites. J.Chem. Pharma. Sci. 11, 39-42.

[15]. ASTM G31-72 (Reapproved 2004): Standard practice for laboratory immersion corrosion testing of metals.

[16]. Liu Zhang sheng, Huang Bo, Shimizu Yoshiaki, Nishimura Toshiyasu, and Matsushima Iwao. 1995. Corrosion resistance of Al-based metal matrix composites. Materials Science Engineering. A198, 113-118.

[17]. Albiter, A. Contreras, A. Salazar, M. Gonzale, and Rodriguez J G. 2006. Corrosion behaviour of aluminium metal matrix composites reinforced with $\mathrm{TiC}$ processed by pressureless melt infiltration. Journal of Applied Electrochemistry. 36 (3), 303-308.

[18]. Jerome,S. Ravisankar, B. Pranab, K M. and Natarajan, S. 2010. Synthesis and evaluation of mechanical and high-temperature tribological properties of in-situ Al-TiC composites. Tribology International. 43, 2029-2036.

[19]. Contreras, A. Leon, C A. Drew, R A L. and Bedolla, E. 2003. Wettability and spreading kinetics of Al and Mg on TiC. Scripta Materialia. 48(12), 1625-1630.

[20]. Muscat, D. Shanker, K. and Drew R A L. 1992. Al/TiC composites produced by melt infiltration. Materials Science and Technology. 8(11), 971-976.

[21]. Ananda Murthy, H C. and Somit Kumar Singh. 2015. Influence of TiC particulate reinforcement on the corrosion behaviour of Al6061 metal matrix composites. Advanced Materials Letter. 6(7), 633-640.

[22]. Alodan, M A. and Smyrl, W H. 1998. Detection of localized corrosion of Aluminium alloys using fluorescence microscopy. Journal of the Electrochemical Society. 145(5), 1571-1577.

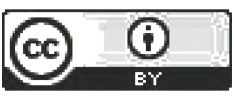

This work is licensed under a Creative Commons Attribution 4.0 International License. 